\title{
Capture rate and representativity of The National Prostate Cancer Register of Sweden
}

Katarina Tomic, Anders Berglund, David Robinson, Marie Hjälm-Eriksson, Stefan Carlsson, Mats Lambe \& Pär Stattin

To cite this article: Katarina Tomic, Anders Berglund, David Robinson, Marie Hjälm-Eriksson, Stefan Carlsson, Mats Lambe \& Pär Stattin (2015) Capture rate and representativity of The National Prostate Cancer Register of Sweden, Acta Oncologica, 54:2, 158-163, DOI: 10.3109/0284186X.2014.939299

To link to this article: https://doi.org/10.3109/0284186X.2014.939299

View supplementary material

曲 Published online: 18 Jul 2014.

Submit your article to this journal $\llbracket$

Џll Article views: 442

View Crossmark data $\asymp$

Citing articles: 24 View citing articles \lceil 


\title{
Capture rate and representativity of The National Prostate Cancer Register of Sweden
}

\author{
KATARINA TOMIC ${ }^{1}$, ANDERS BERGLUND ${ }^{2}$, DAVID ROBINSON ${ }^{1,3}$, \\ MARIE HJÄLM-ERIKSSON ${ }^{4}$, STEFAN CARLSSON ${ }^{5,6}$, MATS LAMBE $^{2,7}$ \& PÄR STATTIN ${ }^{1}$ \\ ${ }^{1}$ Department of Surgical and Perioperative Sciences, Urology and Andrology, Umeå University, Umeå, Sweden, \\ ${ }^{2}$ Regional Cancer Centre, Uppsala University Hospital, Uppsala, Sweden, ${ }^{3}$ Department of Urology, fönköping \\ Hospital, Fönköping, Sweden, ${ }^{4}$ Department of Oncology, Karolinska University Hospital, Stockholm, ${ }^{5}$ Regional \\ Cancer Centre, Stockholm/Gotland, Karolinska University Hospital, Stockholm, ${ }^{6}$ Department of Urology, \\ Karolinska University Hospital, Stockholm and ${ }^{7}$ Department of Medical Epidemiology and Biostatistics, \\ Karolinska Institutet, Stockholm, Sweden
}

\section{ABSTRACT}

Background. Capture rate and representativity of quality registers need to be assessed in order to ensure that register data are generalizable.

Material and methods. In 1998-2009, 103047 men had been diagnosed with prostate cancer and registered in the Swedish Cancer Register to which registration is mandated by law and of these men, $100849 \mathrm{men}(98 \%)$ had also been registered in The National Prostate Cancer Register (NPCR) of Sweden. We compared demographics, cancer treatment, comorbidity, and mortality in men in NPCR, with those who had only been registered in the Cancer Register, by use of data from the Cause of Death Register, the In-Patient Register and the Prescribed Drug Register. In addition, we identified 1929 men who had prostate cancer as underlying cause of death in the Cause of Death Register who had neither been registered in the Cancer Register nor in NPCR.

Results. Compared to men in NPCR, men only registered in the Cancer Register were slightly older, median age 72 versus 71 years, and a lower proportion underwent radical prostatectomy, $15 \%$ versus $27 \%$. Ten year prostate cancer mortality was $23 \%$ (95\% CI 20-25) for men in the Cancer Register only and 24\% (95\% CI 24-25) in NPCR, while mortality from competing causes was $28 \%$ (95\% CI 26-31) and 30\% (95\% CI 30-30), respectively. Men identified with prostate cancer by a death certificate were old and had high comorbidity.

Conclusion. The capture rate of NPCR is very high and there are only modest differences in demographics, cancer treatment, comorbidity, and mortality between the small proportion of men only registered in the Cancer Register and men registered in NPCR, indicating that information in NPCR can be generalized to all men with prostate cancer in Sweden.

Information obtained from high-resolution diseasespecific quality registers are increasingly used for quality assurance including assessment of outcome of various treatments, benchmarking between healthcare providers, and surveillance of adverse events [1]. To ensure that such registers are representative of all cases with the disease, it is important to assess the capture rate of a register and to characterize cases not registered. The National Prostate Cancer Register (NPCR) of Sweden [2] is a cancer quality register to which detailed information on tumor characteristics and primary management in men diagnosed with prostate cancer is reported [3]. The aim of this study was to assess the capture rate and representativity of NPCR by identifying men diagnosed with prostate cancer by the use of other 
register resources and to characterize demographics, cancer treatment, comorbidity, and mortality in these men not registered in NPCR compared to men registered in the NPCR.

\section{Material and methods}

The NPCR of Sweden registers incident cases of prostate cancer (ICD-10 C619, ICD-9 185) in all six health care regions in Sweden since 1998 [4]. Registered variables include diagnostic unit, date of diagnosis, and tumor characteristics according to tumor, node, and metastases (TNM) classification. Other variables include tumor differentiation according to the Gleason classification, serum level of prostate specific antigen (PSA) at diagnosis, and primary treatment delivered within six months after date of diagnosis [3,4]. Cancer registration in Sweden is performed by six Regional Cancer Centers (RCC) and based on two independent reports, one from the responsible clinician who diagnosed the cancer case and one report from the pathologist who made the histopathological examination of the cancer tissue. Each RCC perform coding and logical checks, investigate cases that lack complete clinical, histopathological or cytological data, and transmit data to The Swedish Cancer Register held at The Board of National Health and Welfare (Socialstyrelsen). The RCCs also host 28 cancer quality registers, including NPCR. Data on tumor characteristics, diagnostic work up and primary treatment are extracted from medical charts and entered to NPCR by use of the web-based platform Information Network for Cancer care (INCA) since 2007 [5]. Three forms are used in NPCR: a form for diagnostic data, one form for subsequent primary therapy, and one separate form for radiotherapy. From year 2007 the NPCR diagnostic reporting form is also recognized as the clinical report to the Swedish Cancer Register. Men diagnosed and registered before 1998 in the Cancer Register were excluded from the study group. Men diagnosed with prostate cancer who had been registered in the Cancer Register but not registered in NPCR were identified by use of the individually unique Swedish person identity number in a linkage between the Cancer Register and NPCR. Men registered between 1998 and 2009 with prostate cancer as underlying cause in the Cause of Death Register, but who had not been registered in NPCR or the Cancer Register, i.e. 'death certificate only diagnosis' were identified by cross-linking the Cause of Death Register and the Cancer Register. There is no information transmitted to NPCR or the Cancer Register from the Cause of Death Register so these registers do not include cases based on death certificate only. We retrieved data from the In-Patient
Register, the Prescribed Drug Register, and the Cause of Death Register for men registered in the Cancer Register only and in the NPCR. By use of data on discharge diagnoses in the In-Patient Register up to 10 years prior to the date of diagnosis, we classified cases into four categories of comorbidity according to the Charlson Comorbidity Index (CCI), which is based on discharge diagnoses retrieved from the In-patient Register and is a weighted sum of a number of comorbid factors [6,7]. Data on radical prostatectomy (KEC00, KEC01, KEC10, and KEC20) and bilateral orchiectomy (KFC10 and KFC15) were also obtained from the In-Patient Register [1]. Data on use of GnRH analogs (ATC L02AE) and anti-androgens (ATC L02BB) were retrieved from the Prescribed Drug Register that includes the filled prescription item, amount, and dose from July 2005 and onwards [8]. The date of death and underlying cause were obtained from the Cause of Death Register.

Differences in demographics, cancer treatment, comorbidity, and mortality were examined in men with a prostate cancer diagnosis registered in NPCR, in the Cancer Register only, and in the Cause of Death Register only. For men identified in the Cancer Register only, differences were also studied according to region in which health care was provided. For a subgroup of men in the Cause of Death Register diagnosed in 2006-2009 who had a record of prostate cancer treatment in the Prescribed Drug Register or in the In-Patient Register, the time from start of androgen deprivation therapy to date of death, i.e. time on treatment was used as a proxy for survival time.

\section{Statistical analysis}

Univariable and multivariable logistic regression models with odds ratios and $95 \%$ confidence intervals (CI) were used to investigate the association to registration in NPCR. In a competing risk analysis, prostate cancer mortality and death due to other causes was assessed. Survival time was defined as the time from date of prostate cancer diagnosis to the date of the death, emigration, or end of follow-up on 31 December 2011, whichever event came first. All p-values were two-sided and the level of statistical significance was set to 0.05 . The analyses were performed using R 10.2.

The study was approved by the Research Ethics Board at Umeå University.

\section{Results}

A total of 103047 incident cases of prostate cancer were identified in the Swedish Cancer Register with 
a date of diagnosis between 1998 and 2009. Of these, $100849(98 \%)$ men had also been registered in NPCR. In addition, 1929 men were identified in the Cause of Death Register with prostate cancer as underlying cause of death who had neither been registered in the Cancer Register nor in NPCR.

Table I shows the baseline characteristics for men in NPCR, men in the Cancer Register only, and men identified with prostate cancer by a death certificate only. The median age at diagnosis for men in NPCR was 71 years (25th, 75 th percentile $64-78$ ), and 72 years (25th, 75 th percentile $65-80$ ) for men registered in the Cancer Register only $(\mathrm{p}<0.001)$. The proportion of men in the Cancer Register only increased with older age. The proportion of men with $\mathrm{CCI}=0$ was higher in the Cancer Register only compared to men in NPCR, $74 \%$ versus $65 \%(p<0.001)$. The proportion of men identified with prostate cancer by the Cause of Death Register only who were in the two oldest categories $(77 \%)$ and the proportion of men with high comorbidity (CCI 2 and $3+$ ) $(46 \%)$ was three times higher than for men in the other two groups. A total of 246 men had been registered in NPCR but not in the Cancer Register in 1998-2009 (Supplementary Table I, to be found online at http://informahealthcare.com/doi/abs/ 10.3109/0284186X.2014.939299).
The cancer treatment in the three groups of men diagnosed 2006-2009 is demonstrated in Table II. There were minor differences regarding androgen deprivation therapy between men in NPCR and men in the Cancer Register only, 37\% of cases in NPCR received androgen deprivation therapy versus 31\% for men in the Cancer Register only, out of these $35 \%$ versus $29 \%$ received GnRH analogs, and 32\% versus $26 \%$ received monotherapy with anti-androgens. In analysis of the full study period 1998-2009, the proportion of men who underwent radical prostatectomy was larger in NPCR $(27 \%)$ than in the Cancer Register only (15\%), whereas the 90-day postoperative mortality after radical prostatectomy was quite similar, $0.2 \%$ in NPCR and $0.4 \%$ in the Cancer Register only $(p=0.43)$. The median duration of the perioperative hospital stay was five days for men in NPCR (25th, 75th percentile 3-6) and 4 days (25th, 75th percentile 2-5) for men in the Cancer Register only $(p=0.125)$ and the perioperative hospital stay was less than three days for $14 \%$ of men in NPCR and $27 \%$ of men in the Cancer Register only $(p<0.001)$ (Table III).

There were substantial regional differences in the proportion of men who had only been registered in the Cancer Register, a total of 1455 out of 18147 (7\%) men diagnosed in Stockholm had only been

Table I. Demographics and comorbidity for men diagnosed with prostate cancer and registered in the National Prostate Cancer Register (NPCR) of Sweden, the Cancer Register only (CR Only), and the Cause of Death Register only (DC Only).

\begin{tabular}{lccc}
\hline & NPCR & CR Only & DC Only \\
& $\mathrm{N}=100849$ & $\mathrm{~N}=2198$ & $\mathrm{~N}=1929$ \\
\hline Region & & & \\
$\quad$ Northern & $10376(10 \%)$ & $59(3 \%)$ & $196(10 \%)$ \\
$\quad$ Uppsala Örebro & $21373(21 \%)$ & $135(6 \%)$ & $583(30 \%)$ \\
$\quad$ Western & $21787(22 \%)$ & $207(9 \%)$ & $211(11 \%)$ \\
Southern & $17647(18 \%)$ & $193(9 \%)$ & $374(19 \%)$ \\
Southeastern & $11519(11 \%)$ & $149(7 \%)$ & $159(8 \%)$ \\
Stockholm & $18147(18 \%)$ & $1455(66 \%)$ & $406(21 \%)$ \\
Calendar period & & & \\
$1998-2000$ & $20494(20 \%)$ & $394(18 \%)$ & $444(23 \%)$ \\
$2001-2003$ & $23927(24 \%)$ & $491(22 \%)$ & $498(26 \%)$ \\
$2004-2006$ & $28564(28 \%)$ & $557(25 \%)$ & $459(24 \%)$ \\
$2007-2009$ & $27864(28 \%)$ & $756(34 \%)$ & $528(27 \%)$ \\
$*$ Age at diagnosis, years & & & \\
$<65$ & $31089(31 \%)$ & $593(27 \%)$ & $50(3 \%)$ \\
$66-74$ & $33492(33 \%)$ & $667(30 \%)$ & $158(8 \%)$ \\
$75-79$ & $17244(17 \%)$ & $342(16 \%)$ & $231(12 \%)$ \\
$80-89$ & $17742(18 \%)$ & $519(24 \%)$ & $982(51 \%)$ \\
$90+$ & $1282(1 \%)$ & $77(4 \%)$ & $508(26 \%)$ \\
Charlson Comorbidity Index & & & \\
0 & $65649(65 \%)$ & $1633(74 \%)$ & $630(33 \%)$ \\
1 & $18188(18 \%)$ & $287(13 \%)$ & $423(22 \%)$ \\
2 & $9896(10 \%)$ & $164(8 \%)$ & $226(12 \%)$ \\
$3+$ & $7116(7 \%)$ & $114(5 \%)$ & $650(34 \%)$ \\
\hline
\end{tabular}

*For men diagnosed with prostate cancer in the Cause of Death Register only, date of start of treatment was used as a proxy for date of diagnosis for 698 men who had been diagnosed after 2006, for the remaining 1231 men, date at death was used. 
Table II. Treatment for men diagnosed with prostate cancer in 2006-2009 and registered in the National Prostate Cancer Register, the Cancer Register only (CR Only), and the Cause of Death Register only (DC only).

\begin{tabular}{lccc}
\hline & $\begin{array}{c}\text { NPCR } \\
\mathrm{N}=36967\end{array}$ & $\begin{array}{c}\text { CR Only } \\
\mathrm{N}=1020\end{array}$ & $\begin{array}{c}\text { DC Only } \\
\mathrm{N}=698\end{array}$ \\
\hline $\begin{array}{l}\text { Treatment } \\
\text { *Androgen } \\
\text { deprivation therapy }\end{array}$ & $13691(37 \%)$ & $312(31 \%)$ & $395(57 \%)$ \\
$\begin{array}{l}\text { Radical prostatectomy } \\
* * \text { Other treatments }\end{array}$ & $10111(27 \%)$ & $151(15 \%)$ & $0(0 \%)$ \\
\hline
\end{tabular}

*Information on androgen deprivation therapy (ADT) was retrieved from the Prescribed Drug Register, which started on 1 July 2005. ADT included GnRH analogs and anti-androgens; **other treatments include radiotherapy, conservative treatment (i.e. no active treatment including active surveillance and deferred hormonal treatment), and transurethral resection of the prostate. Treatment had not been registered for 1333 men.

registered in the Cancer Register whereas the corresponding proportion was $0.6 \%$ in the Northern and Uppsala Örebro regions, $0.9 \%$ in the Western region, and $1 \%$ in the Southern and South eastern regions. In a multivariable analysis including calendar period, comorbidity, age at diagnosis, and cancer treatment, the risk of non-registration remained unaltered (data not shown).

There were small differences in mortality between men in NPCR and in the Cancer Register only, the cumulative 10-year prostate cancer mortality was 24\% (CI 95\% 24-25) for men in NPCR and 23\% (CI 95\% 20-25) for men in the Cancer Register only, and the 10-year mortality from competing causes was 30\% (CI 95\% 30-31) for men in NPCR and 28\% (CI 95\% 26-31) for men in the Cancer Register only (Figure 1a and b). As there is no date of diagnosis for men registered with prostate cancer in the Cause of Death Register only, we used start of treatment as proxy for date of diagnosis that was identified in the Prescribed Drug Register for GnRH analogs and anti-androgens as well as in the In-Patient Register for bilateral orchiectomy. The Prescribed Drug Register started in July 2005 so we

Table III. Duration of perioperative hospital stay for radical prostatectomy for 19683 men in the National Prostate Cancer Register, and 262 for men in the Cancer Register only (CR Only) registered 1998-2009.

\begin{tabular}{lrr}
\hline & $\begin{array}{c}\text { NPCR } \\
\mathrm{N}=19683\end{array}$ & $\begin{array}{c}\text { CR Only } \\
\mathrm{N}=262\end{array}$ \\
\hline $\begin{array}{l}\text { *Median stay in days } \\
(25 \text { th-75th percentile })\end{array}$ & $5(3-6)$ & $4(2-5)$ \\
$<3$ days & $2730(14 \%)$ & $71(27 \%)$ \\
$3-5$ days & $10069(51 \%)$ & $136(52 \%)$ \\
$6-7$ days & $4597(23 \%)$ & $33(13 \%)$ \\
$8+$ days & $2287(12 \%)$ & $21(8 \%)$ \\
\hline
\end{tabular}

could assess the date of start of cancer treatment for a subgroup of 698 men who started their treatment after 1 January 2006 by use of the date for the first filled prescription. For men identified with prostate cancer in the Cause of Death Register, median age at start of cancer treatment was 83 years $(25$ th, 75 th percentile 78-88) and median time from start of treatment to death of prostate cancer was 15 months (Figure 1c).

\section{Discussion}

In this population-based study, the capture rate of NPCR was $98 \%$ and there were very modest differences between men registered in NPCR and men in the Cancer Register only regarding demographics, cancer treatment, comorbidity, and prostate cancer and all-cause mortality. These results show that virtually all men diagnosed with prostate cancer are captured in NPCR, that men in the NPCR are representative, and that information in NPCR can be generalized to all men with prostate cancer in Sweden.

Strengths of our study include the use of data from population-based, nationwide health care registries with known high quality [3]. The completeness of the Cancer Register to which registration is mandated by law is very high, although capture has been shown to decrease somewhat with older age [4]. High completeness, between $96 \%$ and $99 \%$, has also been found for other Swedish quality registers, e.g. for cancer of the breast, colon, and rectum [9]. The capture rate of other health care registers used in this study is also high. The Prescribed Drug Register has an extremely high capture rate with less than $0.3 \%$ of all records incomplete due to missing personal identity numbers [10]. The primary discharge diagnosis in the In-Patient Register has been demonstrated to be accurate in $88-90 \%$, although with a wide variation for different diagnoses $[11,12]$. Results from previous studies have shown high accuracy of cause of death for men with prostate cancer in the Cause of Death Register compared to chart reviews $[13,14]$.

Lower capture rate has been noted in private health care, in units with a high case load, and in university hospitals compared to county and local hospitals [4] which may account for the fact that Stockholm, a populous region with many private health care providers and a high proportion of cases treated at large hospitals, had the lowest capture rate.

\section{Demographics}

A patient case file is never closed in NPCR and a small number of cases are entered into the NPCR up to several years after date of diagnosis, which 
(a)

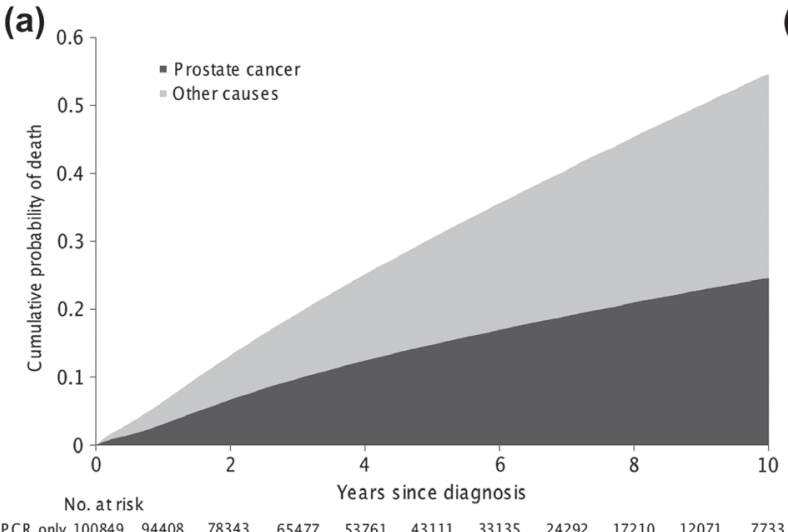

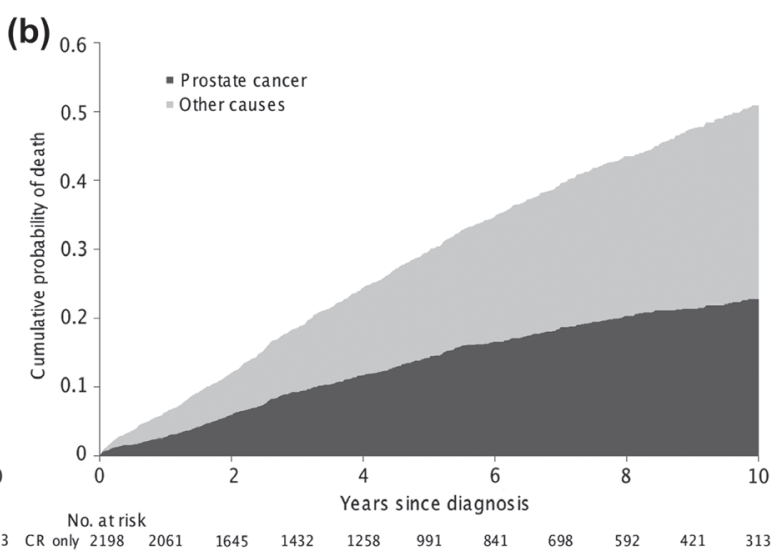

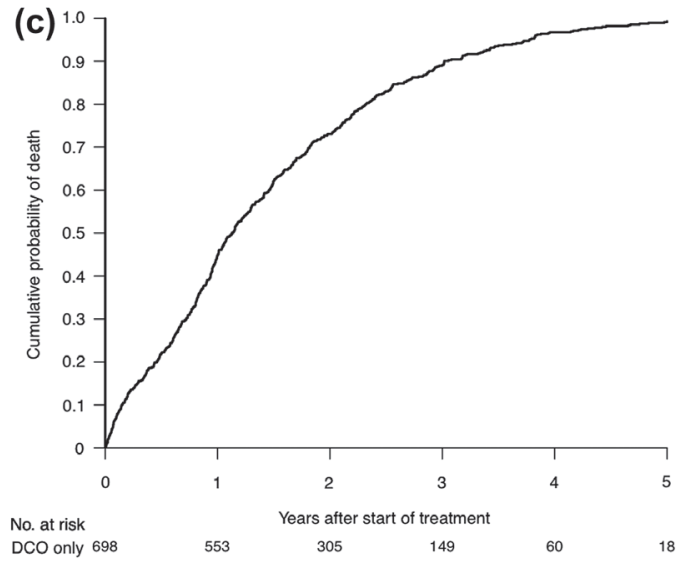

Figure 1. (a) Prostate cancer mortality and mortality from other causes for men diagnosed with prostate cancer and registered in the National Prostate Cancer Register. (b) Prostate cancer and all-cause mortality for men diagnosed with prostate cancer and registered in the Cancer Register only. (c) Prostate cancer mortality for 698 men diagnosed with prostate cancer and registered in the Cause of Death Register only. *Date of start of androgen deprivation therapy: GnRH analogs, anti-androgens, or orchiectomy.

likely explains the slightly lower capture rate in 2007-2009 compared to earlier time periods. Registration decreased with increasing patient age in our study in accordance with previous studies in Norway and Sweden [4].

Men with prostate cancer identified in the Cancer Register only were older, had fewer comorbidities and had approximately half the rate of radical prostatectomy compared to men in NPCR, indicating that these men received less extensive uro-oncological care that likely contributed to the non-registration in NPCR.

\section{Treatment-related factors}

Men not registered in NPCR had a slightly shorter perioperative hospital stay but mortality from prostate cancer as well as from other causes was almost identical for men in NPCR and the Cancer Register only, in contrast to the notion that registration in NPCR would be associated with superior patient care and outcome.

Men diagnosed with prostate cancer as underlying cause of death without a prior diagnosis registered in
NPCR or The Cancer Register during their lifetime were much older at date of death than cases included in the other two registers. These men had a much shorter survival time from start of cancer treatment to death, and this group included a larger proportion of men with a high comorbidity, indicating that this small group consists of frail men diagnosed with advanced prostate cancer at old age.

In conclusion, the capture rate of NPCR was high and there were only modest differences between men in the Cancer Register only and men in NPCR in terms of demographics, cancer treatment, comorbidity, and mortality. Our results indicate that data in NPCR are representative for all men diagnosed with prostate cancer in Sweden and that information in NPCR is generalizable for men diagnosed with prostate cancer in Sweden.

Declaration of interest: This project was funded by the Swedish Research Council 2012-5047, Swedish Cancer Society 110471, and Västerbotten County Council. This project was made possible by the continuous work of the National Prostate 
Cancer Register (NPCR) of Sweden, Pär Stattin (chairman), Anders Widmark, Camilla Thellenberg, Ove Andrén, Anna Bill-Axelson, Ann-Sofi Fransson, Magnus Törnblom, Stefan Carlsson, Marie Hjelm-Eriksson, Bodil Westman, Bill Pettersson, David Robinson, Mats Andén, Jan-Erik Damber, Jonas Hugosson, Ingela Frank-Lissbrant, Maria Nyberg, Göran Ahlgrén, Ola Bratt, René Blom, Lars Egevad, Calle Waller, Olof Akre, Per Fransson, Eva Johansson, Fredrik Sandin, Hans Garmo, Mats Lambe, Karin Hellström, Annette Wigertz, and Erik Holmberg. The authors report no conflicts of interest. The authors alone are responsible for the content and writing of the paper.

\section{References}

[1] Ludvigsson JF, Andersson E, Ekbom A, Feychting M, Kim JL, Reuterwall C, et al. External review and validation of the Swedish national inpatient register. BMC 2011; $11: 450$.

[2] NPCR. Nationella prostatacancerregistret. 2014. [cited 2014 Jun 11]. Available from: http://www.npcr.se.

[3] Van Hemelrijck $M$, Wigertz A, Sandin F, Garmo $H$, Hellstrom K, Fransson P, et al. Cohort profile: The National Prostate Cancer Register of Sweden and Prostate Cancer data Base Sweden 2.0. Int J Epidemiol 2013;42:956-67.

[4] Barlow L, Westergren K, Holmberg L, Talback M. The completeness of the Swedish Cancer Register: A sample survey for year 1998. Acta Oncol 2009;48:27-33.

[5] INCA Sweden. Information network for cancer care 2010. [cited 2014 Jun 11]. Available from: http://www.cancercentrum. se/INCA/kvalitetsregister/.

\section{Supplementary material available online}

Supplementary Table I to be found online at http:// informahealthcare.com/doi/abs/10.3109/0284186X. 2014.939299
[6] Kastner C, Armitage J, Kimble A, Rawal J, Carter PG, Venn S. The Charlson comorbidity score: A superior comorbidity assessment tool for the prostate cancer multidisciplinary meeting. Prostate Cancer P D 2006;9: 270-4.

[7] Charlson ME, Pompei P, Ales KL, MacKenzie CR. A new method of classifying prognostic comorbidity in longitudinal studies: Development and validation. J Chron Dis 1987;40: 373-83.

[8] Wettermark B, Hammar N, Fored CM, Leimanis A, Otterblad-Olausson P, Bergman U, et al. The new Swedish Prescribed Drug Register - opportunities for pharmacoepidemiological research and experience from the first six months. Pharmacoepidem DR S 2007;16:726-35.

[9] Socialstyrelsen. Nationell utvärdering 2013 - Bröst-, prostata-, tjocktarms- och ändtarmscancervård. 2013. [cited 2014 Jun 11]. Available from: http://www.socialstyrelsen.se/ Lists/Artikelkatalog/Attachments/19000/2013-3-8.pdf.

[10] Socialstyrelsen. Socialstyrelsens läkemedelsregister. 2008. [cited 2014 Jun 11]. Available from: http://www.nepi.net/ Socialstyrelsens-laekemedelsregister.htm.

[11] Ingelsson E, Arnlov J, Sundstrom J, Lind L. The validity of a diagnosis of heart failure in a hospital discharge register. Eur J Heart Fail 2005;7:787-91.

[12] Hammar N, Alfredsson L, Rosen M, Spetz CL, Kahan T, Ysberg AS. A national record linkage to study acute myocardial infarction incidence and case fatality in Sweden. Int J Epidemiol 2001;30(Suppl 1):S30-4.

[13] Godtman R, Holmberg E, Stranne J, Hugosson J. High accuracy of Swedish death certificates in men participating in screening for prostate cancer: A comparative study of official death certificates with a cause of death committee using a standardized algorithm. Scand J Urol Nephrol 2011;45:226-32.

[14] Fall K, Stromberg F, Rosell J, Andren O, Varenhorst E. Reliability of death certificates in prostate cancer patients. Scand J Urol Nephrol 2008;42:352-7. 